\title{
INTRODUCTION DE SALMONIDÉS EN MILIEU VIERGE (ÎLES KERGUELEN, SUBANTARCTIQUE) : ENJEUX, RÉSULTATS, PERSPECTIVES.
}

\author{
P. DAVAINE, E. BEALL, \\ avec la collaboration de O. GUERRI et J.M. CARAGUEL.
}

INRA, Station d'Hydrobiologie, B.P. 3, 64310 Saint-Pée-sur-Nivelle, France.

\begin{abstract}
RÉSUMÉ
Les îles Kerguelen (Terres Australes et Antarctiques Françaises) sont, à l'origine, vierges de toute espèce de poisson d'eau douce. Les quelques espèces de Salmonidés, introduites à la fin des années cinquante dans le cadre d'une politique d'occupation et de mise en valeur du Territoire, se sont acclimatées à l'environnement subantarctique et naturalisées avec plus ou moins de succès en fonction de leurs stratégies adaptatives respectives. Objet d'un suivi scientifique continu, ces populations apparaissent comme d'excellents modèles pour des études de génétique et de dynamique des populations. Les phénomènes de colonisation, limités dans un premier temps à l'augmentation régulière des densités de population et une extension rapide intra-rivière, ont connu un développement spectaculaire depuis les années quatre-vingt, à la suite des modifications importantes du climat local, dont l'influence sur les populations a été multiple.
\end{abstract}

La vaste superficie de Kerguelen et les caractéristiques de ses réseaux hydrographiques permettent d'envisager de continuer à tirer parti positivement des introductions passées de Salmonidés, sur les plans scientifique et de mise en valeur du territoire, tout en développant une politique de protection des écosystèmes aquatiques et terrestres conforme à l'évolution actuelle des mentalités.

Mots-clés : acclimatation, salmonidés, subantarctique, colonisation, évolution, changement climatique, cycle vital.

\section{SALMONID INTRODUCTIONS INTO VIRGIN ECOSYSTEMS (KERGUELEN ISLANDS, SUBANTARCTIC) : STAKES, RESULTS, PROSPECTS.}

\section{SUMMARY}

Freshwater fish were, originally, absent from the Kerguelen Islands (French Subantarctic and Antarctic Territories). Some salmonid species, introduced during the late fifties according to a policy of territorial development, acclimatized to the subantarctic environment and became naturalized more or less successfully according to their adaptive strategy. Being scientifically monitored, these populations seem to be excellent models for population genetics and dynamics. The colonization processes, restricted at the beginning to a regular increase in population densities and rapid spreading within the rivers, have dramatically developed since the early eighties, in relation to some important alterations of the local climate which affected populations in many ways.

The vast area of the Kerguelen Islands and the characteristics of their hydrographic systems make it possible to go on exploiting past salmonid introductions for scientific and territorial development, while conducting a policy of aquatic and terrestrial ecosystem conservation consistent with present changes of mentality.

Key-words : acclimatization, salmonids, subantarctic region, colonization, evolution, climatic change, life cycle. 


\section{INTRODUCTION}

La famille des Salmonidés a évolué dans les eaux froides de l'hémisphère nord, colonisant une grande variété de biotopes, soit par la différenciation d'espèces spécialisées comme c'est le cas le long des côtes américaines de l'océan Pacifique (genre Oncorhynchus), soit par le maintien d'un petit nombre d'espèces polymorphes capables de présenter une grande diversité de stratégies adaptatives (genres Salmo et Salvelinus) dans le bassin atlantique. La caractéristique essentielle de la majorité des écosystèmes à Salmonidés étant l'instabilité, les populations ont développé des mécanismes adaptatifs favorisant leur survie dans un environnement fluctuant. Dans une aire géographique donnée, la précision du homing des individus migrateurs et l'isolement reproducteur qui en résulte facilitent, par sélection naturelle, l'adéquation entre les variations périodiques de certains facteurs environnementaux et les exigences de la population lors des phases sensibles de son cycle (ponte, incubation, migrations...). Les opportunités de recroisement à chaque génération entre reproducteurs de stratégies individuelles très variées (sédentaires ou migrateurs d'âges et d'amplitudes de migration différentes...) contribuent à maintenir la variabilité intrapopulation, donc les possibilités de reconstitution des effectifs après des accidents cataclysmiques de courte durée (crues exceptionnelles, sécheresses...). Enfin, à plus long terme, les erreurs de homing et le comportement exploratoire de certains individus permettent de recoloniser de proche en proche des milieux redevenus favorables après extinction de leur population. L'action de ces mécanismes, à différentes échelles de temps, explique l'apparente contradiction entre la rapidité de la recolonisation des régions stérilisées après une glaciation et la spécificité des caractéristiques génétiques de chaque population coadaptée à son environnement.

La répartition géographique actuelle des Salmonidés résulte donc largement des phénomènes de recolonisation naturelle accompagnant la dernière déglaciation (- 13000 ans environ), mais aussi de transferts de populations effectués par l'homme dès la préhistoire (NILSSON, 1972) pour peupler des zones inaccessibles jusqu'alors (parties de bassins versants cloisonnés par des cascades, lacs de montagne, étangs isolés...) et multiplier ainsi ses sources de nourriture. Cette influence anthropique s'est accrue considérablement depuis la seconde moitié du 19ème siècle avec le développement des empires coloniaux, du commerce international, de la technologie et des connaissances sur la biologie des Salmonidés introduits en dehors de leur aire de répartition, dans toutes les parties du monde où les conditions climatiques rendaient leur acclimatation envisageable (ELLIOTT, 1994). A l'heure où la sophistication des techniques permet d'introduire et d'élever des Salmonidés à volonté, la mondialisation de l'économie restreint l'intérêt des introductions dans un but de production à un petit nombre de pays, géographiquement ou socialement favorables; il convient alors de ne plus s'intéresser seulement aux conditions de la réussite de ces introductions, mais aussi à leurs conséquences sur le fonctionnement des écosystèmes ainsi colonisés.

Notre propos est de décrire succinctement les enjeux et les motivations liés aux différentes introductions de Salmonidés dans les eaux douces des îles Kerguelen, d'en résumer les résultats et d'en tracer les perspectives dans un environnement vierge de poissons, a priori hostile, aux limites de l'océan Antarctique.

\section{ENJEUX}

\subsection{Souveraineté}

Découvertes en 1772 par Yves de KERGUELEN qui en prit possession au nom du roi de France, les îles Kerguelen seront rapidement fréquentées par les phoquiers et baleiniers de différentes nationalités, jusqu'à la prise de possession officielle de 1893 et l'octroi de leur concession aux frères BOSSIERE, armateurs du Havre, à charge pour eux d'en assurer la mise en valeur. C'est qu'en effet, depuis le 18ème siècle, le droit des gens ne reconnaît la propriété et la souveraineté d'une nation sur un pays vide que si elle l'occupe réellement et y a formé un établissement dont elle tire un usage actuel (DELÉPINE, 1995). 
En 1924, les îles sont rattachées au Gouvernement général de la France à Madagascar puis, après la faillite des entreprises des frères BOSSIERE et la création en 1951 d'une base permanente, une loi de 1955 les transforme en district du nouveau Territoire d'Outre-Mer des Terres Australes et Antarctiques Françaises chargé d'assurer leur développement.

\subsection{Mise en valeur}

Cette mission de mise en valeur explique l'attitude de l'Administration qui a cherché à " coloniser " l'île, donc à y reconstituer un environnement familier, en acclimatant un grand nombre d'espèces végétales ou animales, domestiques ou non, susceptibles de faciliter la vie des hommes.

C'est dans cet esprit que plusieurs espèces de Salmonidés ont été introduites, à partir de 1955, dans les rivières vierges de poissons, pour le bien-être du personnel des missions pratiquant la pêche sportive et, accessoirement, pour égayer une alimentation basée sur les conserves. Le succès de ces opérations a encouragé le Territoire à tenter l'acclimatation d'espèces plus recherchées et potentiellement plus productives puis, en allant au bout de cette logique, à réaliser une station pilote d'élevage extensif de saumons pour en tester la faisabilité technique, biologique et micro-économique. On est ainsi passé, en quelques années, du simple besoin de lever la frustration de côtoyer des plans d'eau présentant toutes les caractéristiques de rivières à truites, sans les poissons, à l'exploration rationnelle d'une voie de mise en valeur du Territoire par l'exploitation optimisée d'une ressource renouvelable.

\section{RÉSULTATS}

\subsection{Situation biogéographique}

Les îles Kerguelen (6 $500 \mathrm{~km}^{2}$ ) sont situées aux limites de l'océan Antarctique (Fig. 1), ce qui leur confère un environnement marin plus froid que les autres îles subantarctiques, Géorgie du Sud exceptée; au large, la température de la mer en surface varie de $1^{\circ} \mathrm{C}$ en hiver à $5^{\circ} \mathrm{C}$ en été ; la salinité reste élevée de 33,4 à 34,4 pour mille ; les vents d'ouest, quasi constants et très violents, conditionnent la vie animale et végétale sur l'île : insectes aptères ou subaptères, végétation uniquement herbacée. L'intérieur de l'île est composé d'un empilement de coulées basaltiques subhorizontales, profondément entaillées par des vallées en auge, et quelques grandes plaines de dépôts fluvio-glaciaires surtout dans la partie est. Les réseaux hydrographiques sont nombreux et diversifiés : torrents et lacs de montagne, rivières de piémont et de plaines aliuviales, lacs, étangs côtiers, tourbières... Les eaux sont de type oligotrophe, peu minéralisées, avec un équilibre ionique sous la dépendance des aérosols marins (à dominance du sodium sur le calcium et des chlorures sur les carbonates), froides (température moyenne inférieure à $5^{\circ} \mathrm{C}$ pendant 6 à 7 mois par an) et bien oxygénées (100\% de saturation en permanence). Le régime est majoritairement de type pluvial avec des crues fréquentes, un étiage d'été en janvier et février, et de brèves périodes de basses eaux hivernales (pendant les gels prolongés) suivies de débâcles dangereuses. L'origine océanique de l'île, son éloignement de tout continent et sa position légèrement au sud du front polaire expliquent l'absence de toute faune ichtyologique d'eau douce, contrairement aux îles subantarctiques peuplées, toutes situées au nord du front polaire et à proximité d'un continent (Auckland, Campbell). La faune aquatique se limite à quelques espèces abondantes d'Entomostracés planctoniques et benthiques, de petits Oligochètes et Nématodes libres, ainsi qu'à une espèce cosmopolite abondante de chironome (Limnophyes pusillus) présente dans toutes les zones humides. La flore comprend quelques rares Bryophytes et une couverture d'algues vertes filamenteuses pendant la première moitié de l'été.

\subsection{Historique des introductions}

Le temps écoulé depuis les premières introductions peut être divisé en 4 périodes de durées inégales selon les objectifs poursuivis (Tabl. I). 
Tableau I

Découpage de la période d'introductions et de transferts de Salmonidés dans les îles Kerguelen en quatre grandes phases; description des espèces, origines des lots, effectifs, date et mode d'immersion (oeufs embryonnés, alevins nourris, juvéniles, adultes), rivières d'accueil et date de la première reproduction effective.

Table I

Identification of the four main phases during the period of introduction and transplant of Salmonids in the Kerguelen Islands ; species description, origin, numbers, date and planting mode (eyed eggs, fed fry, juveniles, adults), recipient streams and date of the first effective reproduction.

\begin{tabular}{|c|c|c|c|c|c|c|c|c|}
\hline \multicolumn{9}{|c|}{ INTRODUCTIONS ET TRANSFERTS DE POPULATIONS DANS LES ILES KERGUELEN } \\
\hline Phase & Espece & Origine & $\begin{array}{c}\text { Date } \\
\text { expedition }\end{array}$ & $\begin{array}{l}\text { Efrectur } \\
\text { immerge }\end{array}$ & Stade & $\begin{array}{c}\text { Date } \\
\text { introduction }\end{array}$ & $\begin{array}{c}\text { Riviteres } \\
\text { emporissonneess }\end{array}$ & $\begin{array}{l}\text { Annee de lere } \\
\text { reproduction }\end{array}$ \\
\hline $\begin{array}{c}1010 \\
1955-1962\end{array}$ & $\begin{array}{l}\text { Salmo trutta } \\
\text { Salvolinus tomennatis } \\
\text { Salvelinus namaycush } \\
\text { Oncomynchus mykiss }\end{array}$ & 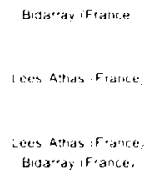 & 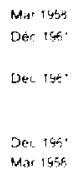 & 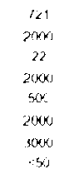 & 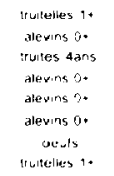 & 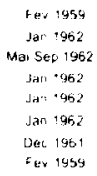 & $\begin{array}{l}\text { Riv. Studer } \\
\text { Riv. Studer } \\
\text { Riv. du Chstosu } \\
\text { Riv. Studer } \\
\text { Riv. du Chsteau } \\
\text { Riv du Sud } \\
\text { Riv. Studer } \\
\text { Riv Studer }\end{array}$ & 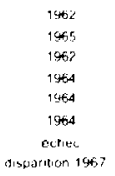 \\
\hline $\begin{array}{c}\text { Zerne } \\
1963.1974\end{array}$ & $\begin{array}{l}\text { Sajmo trufte } \\
\text { Salvalinus fontinalis }\end{array}$ & 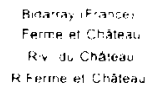 & $\begin{array}{l}\text { Det } 197 / \\
\text { transfert } \\
\text { transtert } \\
\text { transtert }\end{array}$ & $\begin{array}{l}1,31 \\
5,3 \\
51 \\
6 n\end{array}$ & $\begin{array}{l}\text { alevins c. } \\
\text { Iruses } \\
\text { ollewitis u. } \\
\text { armbles }\end{array}$ & $\begin{array}{l}\text { Aur } 1973 \\
\text { Aur } 1974 \\
\text { Mor } 19 / 2 \\
\text { Ayr } 1974\end{array}$ & $\begin{array}{l}\text { Riv du Borgne } \\
\text { Etang de la Sabliers } \\
\text { Rly du Borgne } \\
\text { Etang de la Sablierre }\end{array}$ & $\begin{array}{c}-977 \\
.975 \\
\text { Deds } 90 \text { ret } \\
4975\end{array}$ \\
\hline $\begin{array}{c}38 \mathrm{ma} \\
1975-1981\end{array}$ & $\begin{array}{c}\text { Saimo trutta } \\
\text { Salmo satar } \\
\text { Oncorhymchus kisuteh }\end{array}$ & 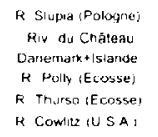 & 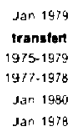 & $\begin{array}{r}23000 \\
10000 \\
105954 \\
70500 \\
99000 \\
5000\end{array}$ & 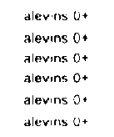 & $\begin{array}{l}\text { Mar 1979 } \\
\text { Dec } 1981 \\
1975-1979 \\
1977+1978 \\
\text { Avr } 1980 \\
\text { Mar } 1978\end{array}$ & $\begin{array}{l}\text { Riv. de I' Acaena } \\
\text { Riv. du Nord } \\
\text { Riv. des Korrigans } \\
\text { Riv. Armor } \\
\text { Riv. Armor } \\
\text { Riv. Grisanehe }\end{array}$ & $\begin{array}{l}1983 \\
1986 \\
1981 \\
189 ? \\
1986 \\
1980\end{array}$ \\
\hline $\begin{array}{c}\text { 4eme } \\
1982.1992\end{array}$ & 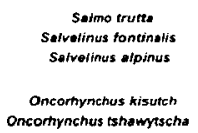 & 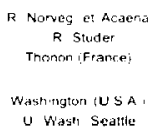 & $\begin{array}{l}\text { transtert } \\
\text { transtert } \\
\text { Dec 1997 } \\
1994.1999 \\
\text { Dec } 1996\end{array}$ & $\begin{array}{c}1000 \\
284 \\
20000 \\
269 \\
275508 \\
60000\end{array}$ & 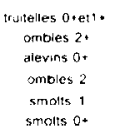 & $\begin{array}{l}\text { Fer 1991 } \\
\text { Dec 1992 } \\
\text { AOs } 1991 \\
\text { Dec } 1992 \\
1985-1991 \\
\text { Jul } 1987\end{array}$ & $\begin{array}{l}\text { Riv. Armor } \\
\text { Riv. Armor } \\
\text { Lac des Fougeres } \\
\text { Riv. Armor } \\
\text { Riv. Armor } \\
\text { Riv. Armor }\end{array}$ & $\begin{array}{c}1993 \\
1993 \\
\text { Das de rep } \\
\text { pas de rep } \\
1987 \\
\text { pas de rep }\end{array}$ \\
\hline
\end{tabular}

La 1ère phase, de 1955 à 1962 ( 8 ans), couvre toutes les premières tentatives d'introductions qui ont donné lieu à l'immersion d'œufs, d'alevins ou de truitelles. Les difficultés principales ont consisté à faire coïncider l'état de maturité et le développement des oeufs avec la date de départ et la durée du voyage du cargo effectuant la relève des missions, puis à maîtriser les techniques de repeuplement pour pallier l'effet des crues sur les oeufs immergés à contre-saison. Seuls les déversements d'alevins et de juvéniles ont été efficaces. A la fin de cette période, il y avait de la truite commune (Salmo trutta) dans la rivière Studer et du Château, et de l'omble de fontaine (Salvelinus fontinalis) dans les rivières Studer, du Sud et du Château; des juvéniles de truite arc-en-ciel (Oncorhynchus mykiss), déversés dans la rivière Studer, ont donné lieu à quelques recaptures jusqu'en 1967 sans qu'il y ait eu de reproduction (Tabl. I et Fig. 1). La rivière de la Ferme, petit ruisseau de la base de Port aux Français, auprès de laquelle étaient situées les installations d'incubation, a également reçu des truites et des ombles, mais n'est pas mentionnée dans le tableau car trop petite et trop perturbée pour accueillir un peuplement stable. Pour plus de détails, on consultera RICHERT (1958), LÉSEL et al. (1971), DAVAINE et BEALL (1982 a), DAVAINE (1989).

La 2ème phase, de 1963 à 1974 (12 ans), a été consacrée au recueil de données sur les populations en cours de naturalisation, à l'exclusion de nouvelles introductions. On mentionnera cependant les quelques alevins de truite, excédent d'une introduction à Crozet en 1972, apportés à Kerguelen et immergés dans la rivière du Borgne où ils feront souche. Les seuls mouvements de populations concernent les transferts locaux ayant pour but d'offrir aux hivernants des lieux de pêche à proximité de la base, comme dans le cas de l'étang de la Sablière, seul à maintenir un peuplement équilibré de truites et d'ombles. 


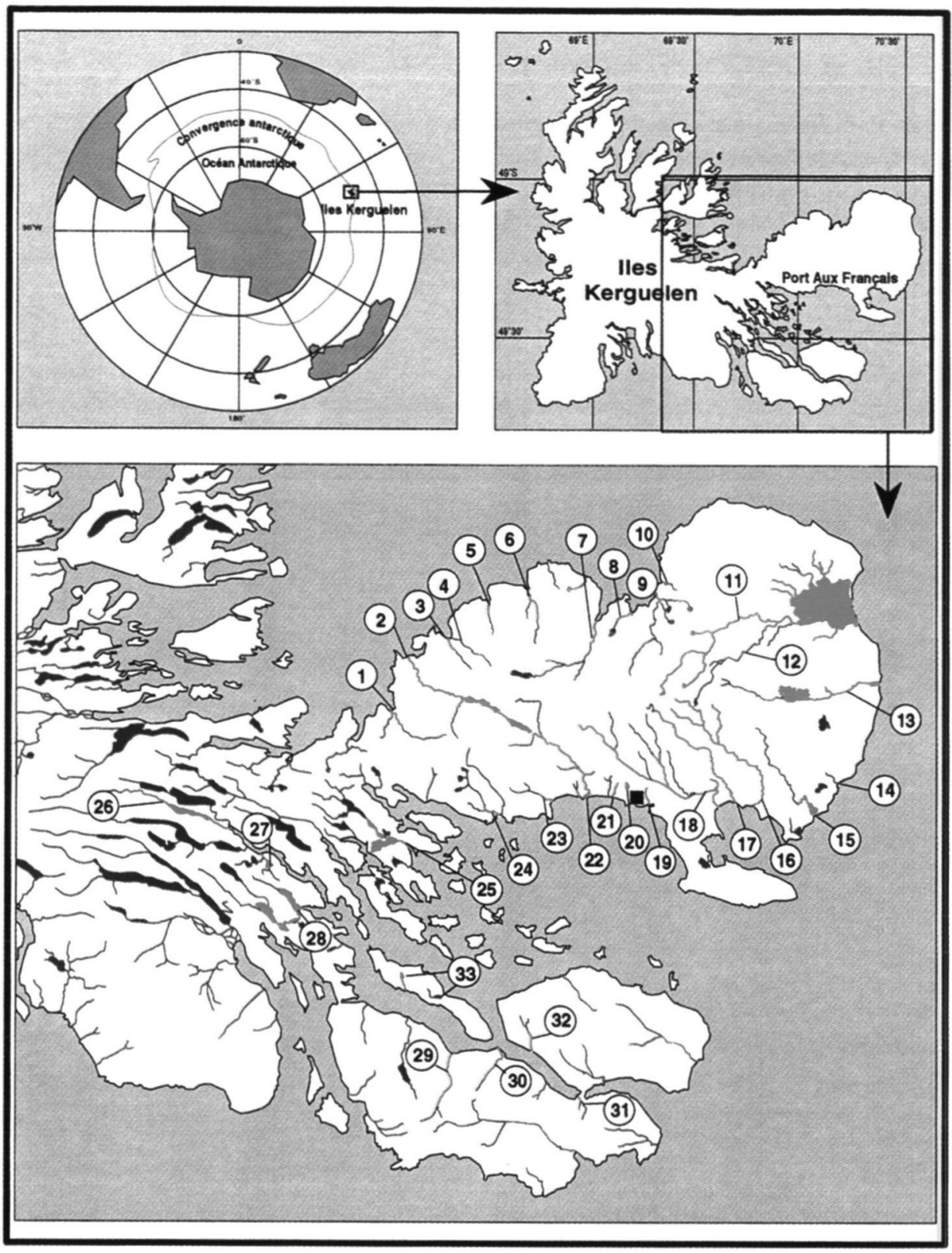

Figure1

Carte de situation des îles Kerguelen et des rivières peuplées en Salmonidés.

Figure 1

Situation map of the Kerguelen Islands and of rivers populated with Salmonids.
1. R. du Val de l'Ouest
12. R. du Volcan
23. R. des Américains
2. R. Studer
13. R. des Manchots
3. R. du Doute
14. R. des Calcédoines
4. R. du Charbon
15. R. du Bungay
5. R. du Sérail
16. R. des Albatros
6. R. des Chasseurs
17. R. Norvégienne
7. R. du Nord
18. R. du Château
24. R. des 3 Lacs
25. R. des Korrigans
26. R. Grisanche
27. Lac des Fougères
28. R. d'Armor
8. R. des Pépins
19. R. de la Ferme
9. R. des Cataractes
20. Etang de la Sablière
10. R. des Hautes Mares
21. R. du Borgne
29. R. de l'Acaena
30. R. de la Planchette
31. R. du Val Raide
11. R. de l'Est
22. R. du Sud
32. R. des Mouettes
33. Etangs de l'lle Longue 
Pendant la 3ème phase, de 1975 à 1981 (7 ans), les introductions ont repris dans le cadre d'expériences contrôlées avec diverses souches de saumon atlantique (Salmo salar), une souche polonaise de truites de mer de la Baltique ( $S$. trutta), réputée pour sa forte croissance, et du saumon coho ( $O$. kisutch), importé des USA. Toutes ces nouvelles introductions ont été effectuées dans des rivières éloignées des précédentes, de façon à éviter les interactions entre populations. Une opération de transfert a concerné la descendance d'un couple de truites de mer originaires de la rivière du Château, homozygotes pour un gène rare, lâchée dans la rivière du Nord. La particularité génétique de cette souche permettra de déterminer ultérieurement l'origine du peuplement éventuel des rivières voisines et la vitesse de colonisation.

La 4ème phase, de 1982 à 1992 (11 ans), est concernée par la conception et la réalisation de l'expérience pilote de pacage marin dans le réseau hydrographique d'Armor (DAVAINE, 1988) ; les espèces utilisées ont été les saumons coho et chinook (O. tshawytscha) et, pour la partie élevage en eau douce, les souches locales de truite commune des rivières Norvégienne et de l'Acaena, les ombles de fontaine de la rivière Studer, ainsi qu'une souche introduite d'omble chevalier (Salvelinus alpinus) originaire du lac Léman. A la fin de l'expérience, beaucoup de ces poissons ont été libérés dans le lac d'Armor sans qu'il soit encore possible de statuer sur leur naturalisation définitive.

\subsection{Structure génétique et stratégies adaptatives}

Sur les quatre espèces introduites pendant la première phase, deux seulement se sont naturalisées : la truite commune et l'omble de fontaine, colonisant des biotopes divers (étangs, lacs, rivières, baies marines) en manifestant une grande variabilité morphologique et comportementale (forme de rivière sédentaire, forme marine migratrice, forme lacustre).

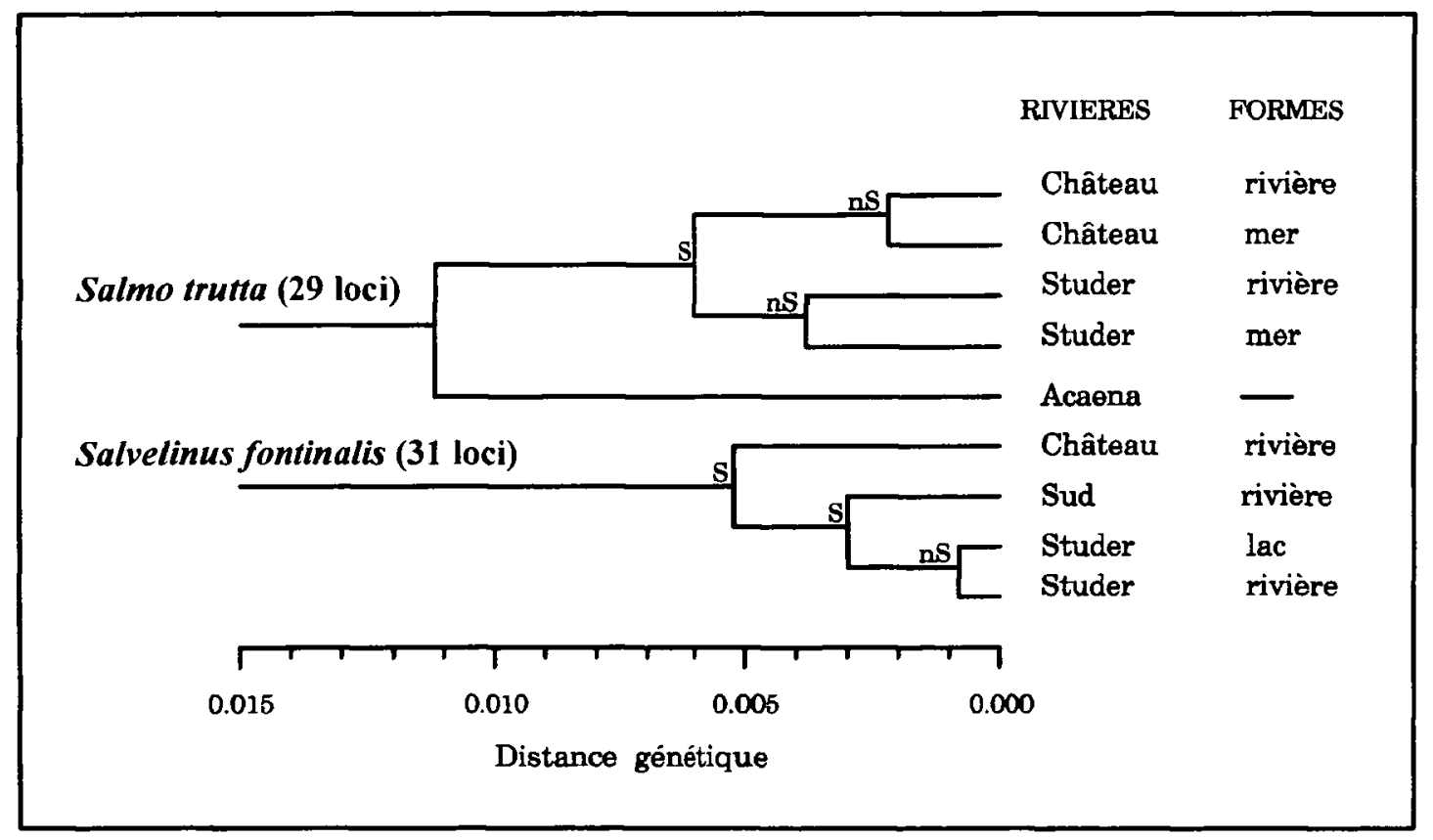

\section{Figure 2}

Dendrogramme des distances génétiques entre sous-populations de truite commune et d'omble de fontaine. $S$ : significatif ; nS : non significatif (d'après GUYOMARD et al., 1984).

\section{Figure 2}

Genetic distances dendrogram for subpopulations of brown and brook trout. $\mathbf{S}$ : significant ; nS : non significant (from GUYOMARD et al., 1984). 
L'exemplarité de ces populations tient au fait qu'elles proviennent, pour chaque espèce, d'un pool génique unique introduit dans différents réseaux hydrographiques : une seule expédition pour l'omble de fontaine, deux expéditions à partir de la même pisciculture pour la truite commune (Tabl. I). Elles constituent donc un cas particulièrement intéressant d'étude de la relation entre différenciation génétique et différenciation en formes que l'on a élevées parfois au rang de sous-espèces. L'analyse électrophorétique de 14 systèmes enzymatiques correspondant à 31 loci, réalisée en 1981 sur des échantillons de ces sous-populations a démontré qu'il n'y avait pas de différence génétique significative entre formes d'une même rivière (GUYOMARD et al., 1984). Ainsi, à partir d'un même pool initial, les dendrogrammes des distances génétiques regroupent, en premier lieu, les différentes formes prélevées dans un même réseau hydrographique (Fig. 2) qui semblent donc résulter d'une variabilité comportementale au sein de populations panmictiques. Par contre, entre rivières isolées géographiquement, des divergences génétiques significatives apparaissent, en l'espace de 5 à 6 générations au plus, soit sous l'action de pressions de sélection différentes entre milieux, soit, plus probablement, par dérive génétique provenant des mortalités aléatoires ou des difficultés de rencontre parmi un effectif reproducteur relativement faible pendant la première phase de colonisation. Enfin, les taux d'hétérozygotie élevés dans chaque sous-population et leur homogénéité remarquable témoignent d'une bonne conservation de la variabilité génétique initiale qui exclurait un effet fondateur sur la variabilité intra-population.

Les études écologiques entreprises depuis 1970 ont permis de préciser les comportements reproducteurs, alimentaires, migratoires et la cinétique démographique des populations en place, d'éclaircir certains aspects de leur dynamique, et de décrire quelques stratégies utilisées par les truites et les ombles pour s'adapter et se développer efficacement dans l'environnement subantarctique (DAVAINE et BEALL, 1982 a et b ; THOMAS et al., 1981 ; DAVAINE, 1988 ; DAVAINE et BEALL, 1992). Compte tenu du caractère récent de l'introduction, le terme de stratégie adaptative ne recouvre pas, dans le cas présent, le produit d'une évolution in situ par sélection naturelle, mais plutôt des ajustements à court terme, témoignant d'une grande plasticité phénotypique autorisée par la variabilité génétique des populations initiales. "De tels phénomènes, dont la valeur adaptative est évidente, ne peuvent toutefois pas être exclus de l'étude des stratégies adaptatives " (BARBAULT et BLANDIN, 1979).

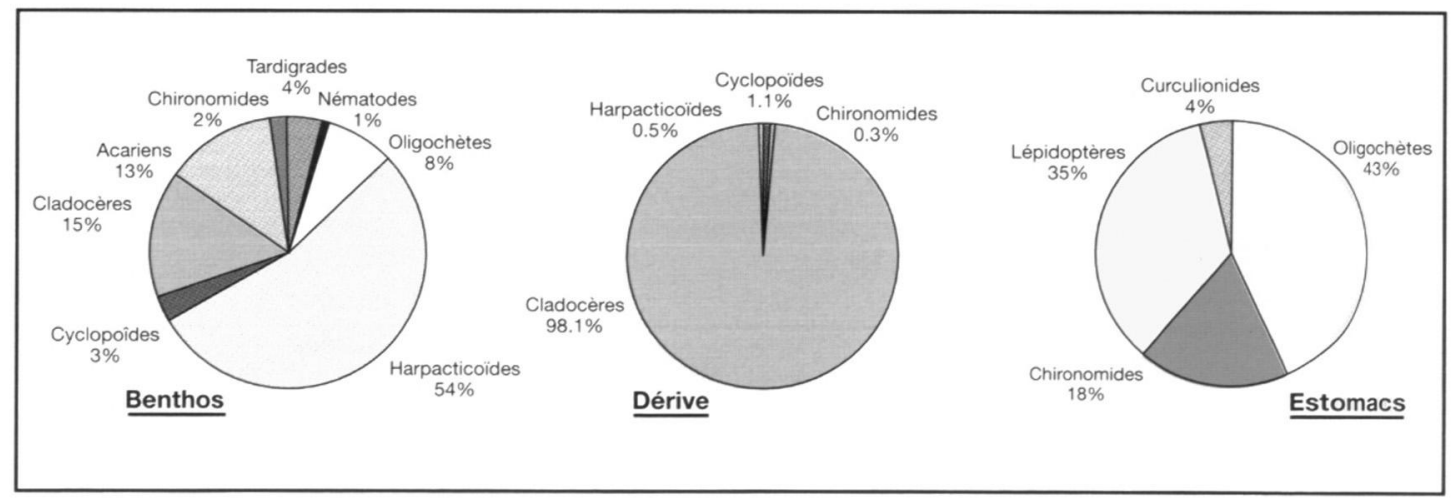

Figure 3

Comparaison des fréquences en nombre des différents groupes faunistiques entre la faune benthique, la faune en dérive et les contenus stomacaux de truites, pendant un cycle de $24 \mathrm{~h}$ en période hivernale (d'après WOJTENKA et VANSTEENBERGHE, 1982). On remarque que la majorité des proies ingérées sont d'origine terrestre rivulaire.

Figure 3

Comparison of number frequencies of different faunistic taxa among benthic fauna, drift fauna and stomach contents of brown trout, during a winter $24 \mathrm{~h}$-cycle (from WOJTENKA and VANSTEENBERGHE, 1982). Note that most ingested preys are of terrestrial origin. 
Les deux principaux facteurs environnementaux limitant la croissance et la production des Salmonidés dans les eaux douces de Kerguelen sont, d'une part, les basses températures et la brièveté de la saison favorable (environ 3 mois d'été) et, d'autre part, la faible diversité, la petite taille et la mauvaise accessibilité des proies (Fig. 3). Par contre, les eaux marines côtières, en particulier l'espace infralittoral, abritent une biomasse très importante d'invertébrés accessibles et de taille adaptée aux besoins des Salmonidés. Dans ces conditions, l'omble de fontaine s'est mieux adapté aux eaux douces, lacs et rivières, que la truite commune (DAVAINE et BEALL, 1982 a) : sa vitesse de croissance est supérieure aux basses températures, sa ponte au début de l'automne permet aux alevins d'émerger des graviers et de prendre territoire dès le début de l'été, bénéficiant ainsi d'une taille supérieure et de l'antériorité de résidence par rapport à la truite, avantage qui se poursuit pendant les deux premières années de vie en rivière. Ultérieurement, les ombles adultes filtrent plus efficacement le zooplancton de petite taille que les truites et, à longueur égale, leur ouverture buccale leur donne accès à des proies d'origine exogène plus grandes (insectes terrestres, araignées, mollusques...). En outre, les ombles arrivent à maturité un an avant les truites sédentaires et deux ans avant les femelles migratrices, ce qui a favorisé le développement de leurs populations pendant la première phase de colonisation. C'est ainsi que, dans le déversoir du lac Studer, en amont d'une cascade infranchissable, les ombles de lac qui s'y reproduisent se sont rapidement développés en supplantant la population de truites entre 1974 et 1980 , puis, à la suite d'une augmentation très sensible de la pression de pêche, les biomasses des deux espèces sont un peu rééquilibrées en 1994, en raison de la plus grande vulnérabilité des ombles à l'exploitation (Tabl. II).

\section{Tableau II}

Evolution comparée des biomasses de truites et d'ombles dans le déversoir du lac Studer (populations estivales de juvéniles et d'adultes sédentaires issus de la reproduction des adultes de lac).

\section{Table II}

Changes in brown and brook trout biomass in the outlet of Studer Lake (summer populations of sedentary juveniles and adults coming from the reproduction of lake adults).

\begin{tabular}{|c|ccc|}
\hline \multirow{2}{*}{ ESPÈCE } & \multicolumn{3}{|c|}{ BIOMASSE KG/HA } \\
\cline { 2 - 4 } & 1974 & 1980 & 1994 \\
\hline TRUITE & 15 & 8 & 37 \\
OMBLE & 10 & 81 & 58 \\
\hline
\end{tabular}

Malgré une moins bonne adaptation aux basses températures, la truite commune présente une stratégie adaptative de type arctique, très efficace dans les rivières accessibles par la mer, combinant une migration trophique estivale en zone littorale avec un retour systématique en eau douce pendant la saison froide pour échapper aux conséquences du couple salinité-température très défavorable (Fig. 4). L'extraordinaire abondance des proies (essentiellement des Amphipodes) de l'étage infralittoral se traduit par des croissances marines fortes (Fig. 5a), en dépit des températures estivales basses $\left(6\right.$ à $7,5^{\circ} \mathrm{C}$ ) dans les baies intérieures. Le faible impact des prédateurs sur des poissons de grande taille et l'abri des herbiers géants à macrocystis favorisent la survie des truites migratrices qui sont par ailleurs protégées contre une pression de pêche excessive, dès leur retour en rivière, par une réglementation appropriée. Dans ces conditions, la forme migratrice de la truite commune tend à constituer l'essentiel de la biomasse de l'espèce (THOMAS et al., 1981) et de son recrutement, compte tenu d'une fécondité très élevée (Fig. 5b). La fraction sédentaire contribue peu au recrutement annuel, car les femelles y sont minoritaires et leur sous-alimentation 


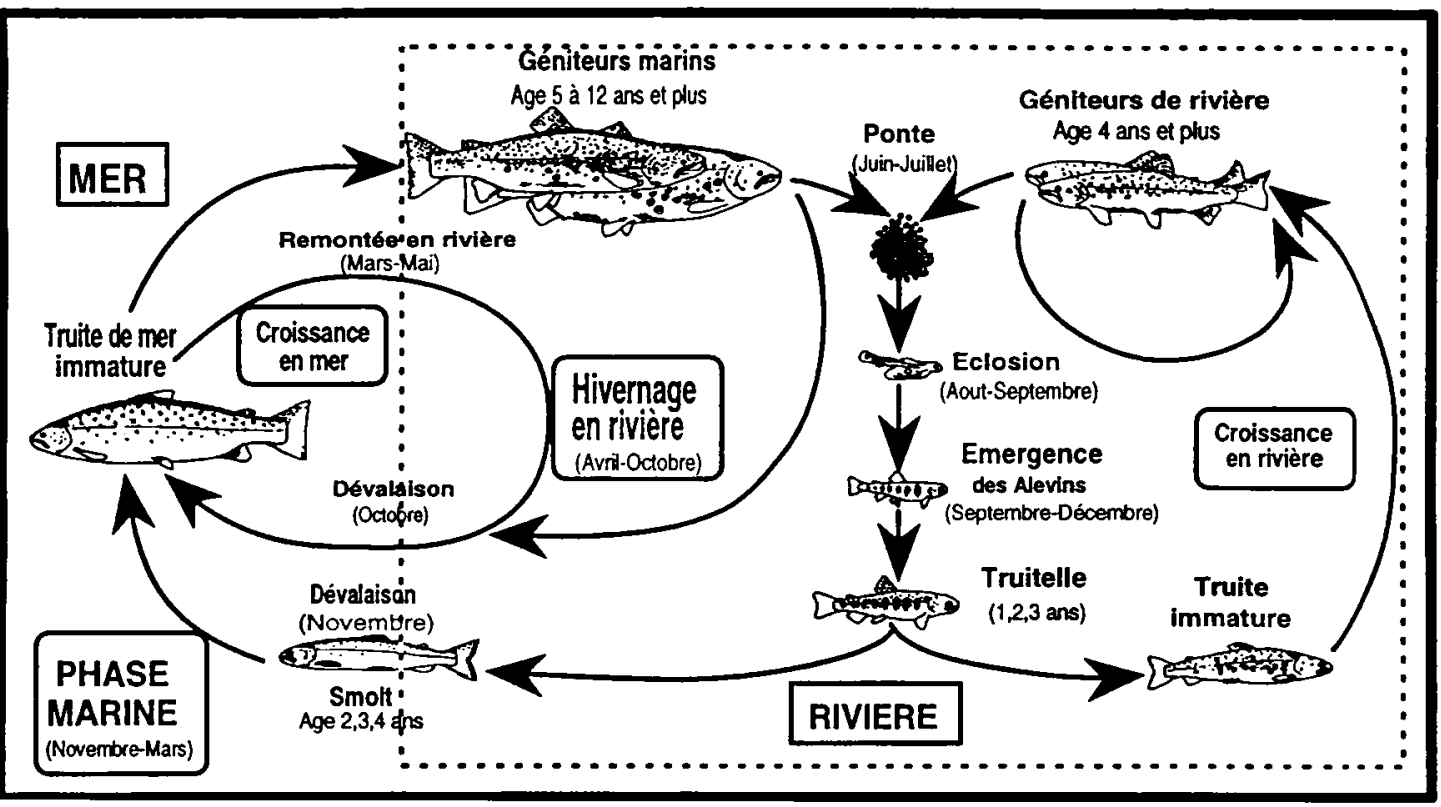

Figure 4

Cycle vital de la truite commune à Kerguelen (formes migratrice et sédentaire).

Figure 4

Life cycle of the migratory and resident brown trout in Kerguelen.

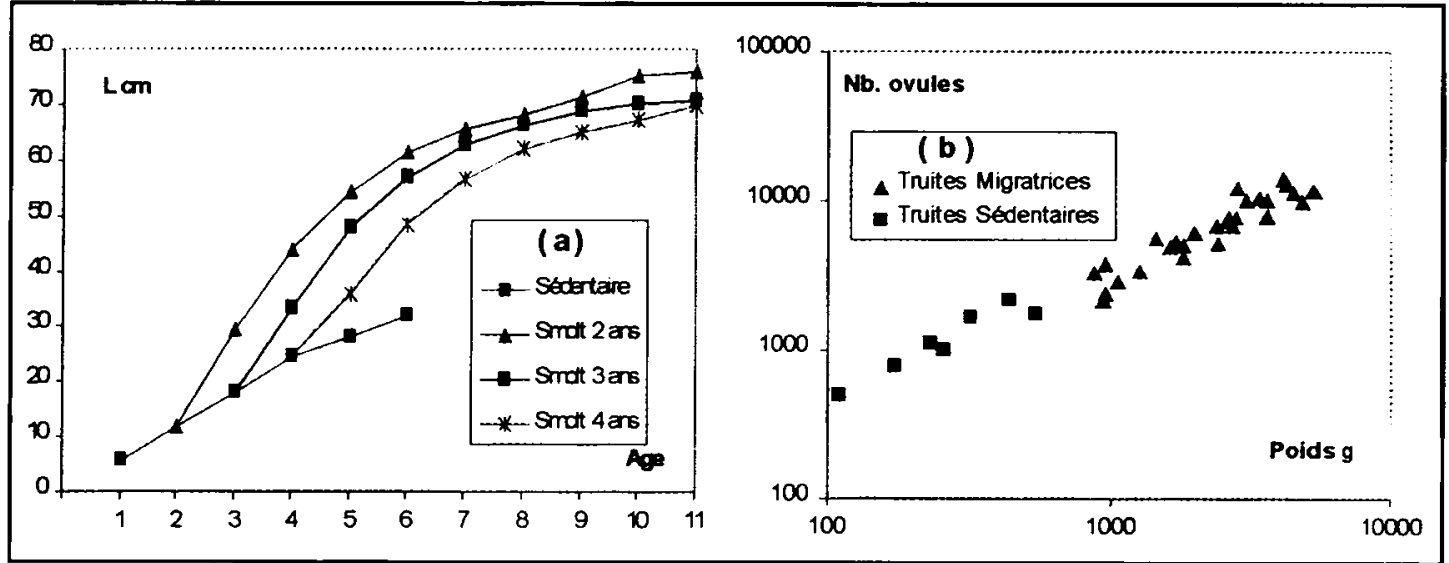

Figure 5

a - Courbes de croissance (moyennes des longueurs à la fourche) des truites sédentaires et des truites migratrices ayant smoltifié à 2, 3 ou 4 ans (échantillon de 3153 truites, tous âges confondus).

b - Fécondité (nombre d'ovules) en fonction du poids corporel des femelles sédentaires et migratrices de truite commune.

Figure 5

a - Growth curves (mean fork lengths) of sedentary brown trout and of 2, 3 or 4 years old smolt of migratory trout (sample of 3153 various age trout).

b - Fecundity (ova number) as a function of body weight of sedentary and migratory brown trout females. 
chronique ne permet pas de survivre à plus d'une, voire deux reproductions consécutives; elle se maintient néanmoins, puisque les plus grands mâles peuvent frayer avec des femelles migratrices et que, dans la descendance d'un couple de truites de mer, on retrouve toujours une proportion non négligeable d'individus sédentaires, comme l'atteste la structure des populations des rivières colonisées naturellement par la reproduction de truites de mer vagabondes.

Dans la rivière Norvégienne, en 1979, la production nette de la population de truites juvéniles et de sédentaires était de $22 \mathrm{~kg}$ par hectare et par an, alors que celle des truites de mer, ramenée à la surface productrice de la rivière, était de $100 \mathrm{~kg}$ par hectare et par an. En dépit d'une meilleure adaptation aux eaux douces, la population d'ombles de cette rivière n'a pu se développer face à la concurrence des truites migratrices qui saturent les zones de frayères et de production de juvéniles, et en raison d'une pression excessive de la pêche estivale qui les atteint plus gravement que les truites sédentaires. Contrairement à la situation du déversoir du lac Studer, les ombles de la rivière Norvégienne sont au bord de l'extinction en 1994 (Tabl. III).

\section{Tableau III}

Biomasses et densités (en alevins et pour le reste de la population) de truites et d'ombles dans la rivière Norvégienne, durant l'été 1994.

Table III

Biomasses and densities (for fry and for the rest of the population) of brown and brook trout in the Norvégienne river, during the summer 1994.

\begin{tabular}{|c|c|c|c|}
\hline ESPÈCE & BIOMASSE KG/HA & $\begin{array}{c}\text { DENSITÉ N/HA } \\
\text { (SANS ALEVINS) }\end{array}$ & $\begin{array}{c}\text { DENSITÉ N/HA } \\
\text { ALEVINS }\end{array}$ \\
\hline TRUITE & 139.3 & 2850 & 14430 \\
OMBLE & 3.5 & 40 & 20 \\
\hline
\end{tabular}

Malgré une vitesse de croissance rapide, la brièveté du séjour en mer limite la croissance annuelle, et les fortes tailles atteintes par les reproducteurs ( 6 à $10 \mathrm{~kg}$ de poids corporel) sont le résultat d'une bonne survie et de l'enchaînement d'un grand nombre de migrations marines consécutives (10 et plus). Dans le but d'améliorer ces performances, une expérience d'introduction de truites de mer de la Baltique (rivière Slupia, Poméranie), à forte croissance et grande amplitude de migration, a été tentée en 1979 dans la rivière de l'Acaena (Tabl. I et Fig. 1). Même si, après trois générations, cette naturalisation est un succès, cette population présente les mêmes caractéristiques et les mêmes performances vis-à-vis du milieu subantarctique que la souche originaire d'une pisciculture du pays basque précédemment naturalisée, montrant ainsi l'influence déterminante de l'environnement dans le choix des stratégies comportementales.

Le même type d'expérience réalisé avec différentes souches de saumon atlantique (une danoise, une islandaise, deux écossaises ; Tabl. I et Fig. 1), plus ou moins adaptées aux rivières froides et de taille modeste, a révélé l'excellente survie en eau douce des alevins relâchés dans des rivières vierges de poissons, une croissance supérieure à celle des truites, une smoltification et un départ en mer normaux. Malheureusement, les quelques retours d'adultes sont restés exceptionnels et les populations ne se sont maintenues que grâce à la reproduction de géniteurs sédentarisés en lac, conformément à d'autres tentatives d'introduction dans l'hémisphère sud de cette espèce, qui n'a encore jamais présenté de stock migrateur naturalisé en dehors de son aire d'origine (THORPE, 1980). Par contre, un essai limité réalisé en 1978 avec une espèce du Pacifique réputée pour sa plasticité, le saumon coho, a donné immédiatement 
d'excellents résultats : smoltification à un an, bonne survie en mer, ponte avant trois ans, pas de sédentarisation en lac.

La stratégie développée par le coho est intermédiaire entre celles du saumon atlantique et de la truite. On peut penser que, migrateur de plus faible amplitude, souvent inféodé au plateau continental, il évite les erreurs de navigation et/ou les mortalités par prédation excessive chez le saumon atlantique, peu apte aux transplantations en dehors de son aire d'origine. Supportant mieux les salinités élevées aux basses températures, le coho hiverne en mer et retourne en rivière après un séjour de 16 à 18 mois, voire 28 mois, à une taille très supérieure à celle des truites de mer après deux cycles migratoires. Cependant, cet hivernage en mer couplé à un cycle court, qui s'achève par la mort obligatoire après la ponte, et une grande homogénéité comportementale des populations rendent le saumon coho très vulnérable à l'instabilité de son environnement. C'est ainsi qu'après l'hiver particulièrement froid de 1988, aucun saumon n'est revenu en rivière au printemps suivant. Ce sont les caractères de bonne survie, cycle court et vitesse de croissance, qui ont fait choisir le coho pour l'expérience pilote de pacage marin de saumons à partir des installations d'élevage du lac d'Armor. Malgré la démonstration de la faisabilité biologique et technique de ce projet (DAVAINE, 1991), il n'eut pas de suite après 1992, en raison d'un contexte économique défavorable.

Une dernière espèce, l'omble chevalier, a été introduite en 1991. Testée dans le cadre des élevages à la station d'Armor, elle est supérieure à toutes les autres espèces de Salmonidés pour sa vitesse de croissance en eau froide, mais il est peu probable que cette souche d'origine lacustre (lac Léman) puisse exploiter le milieu marin et se développer comme la truite commune, qui est sans conteste l'espèce la mieux adaptée aux conditions environnementales générales des îles Kerguelen.

\subsection{Facteurs climatiques et dynamique de la colonisation}

\subsubsection{Evolution climatique}

Au cours de la période d'acclimatation des Salmonidés à Kerguelen, la température moyenne annuelle de l'air, enregistrée à la station météorologique de Port aux Français, est passée par un minimum au début des années soixante pour augmenter régulièrement, et d'une façon très significative, jusqu'à la fin des années quatre vingt (DAVAINE et BEALL, 1992). Si l'on regroupe ces données pour les phases définies précédemment, on constate que l'augmentation de $1^{\circ} \mathrm{C}$ concerne les deux dernières phases et que cela va de pair avec l'augmentation des précipitations annuelles (Fig. 6a).

Cette évolution, qui se concrétise par un recul important des glaciers et la disparition des névés de basse altitude, relève d'un phénomène plus général déjà décrit par SALINGER (1982) en Nouvelle-Zélande pour la période 1950-1975, puis par CHOWN et SMITH (1993) pour l'île subantarctique de Prince Edward où certaines chaînes trophiques s'en trouvent modifiées. De tels changements ont des répercussions écologiques d'autant plus marquées que les écosystèmes concernés, antarctiques et subantarctiques, ont des équilibres fragiles (YOUNG, 1991).

\subsubsection{Evolution des populations intra-rivière}

Lorsque les habitats étaient favorables, la colonisation des rivières a été extrêmement rapide, aussi bien vers l'amont que vers l'aval, selon le lieu d'introduction des poissons pendant la 1ère phase. Cependant, une zone défavorable de rapides à forte pente sur plus d'un kilomètre était suffisante pour bloquer la colonisation vers l'amont, comme l'ont montré les observations systématiques le long des rivières.

A partir de la 2ème phase, la densité de population a augmenté exponentiellement dans les zones peuplées (Fig. $6 \mathrm{~b}$ ) jusqu'à atteindre le niveau inattendu des bonnes rivières à truite européennes, dont les ressources trophiques sont incomparablement plus élevées.

Les croissances individuelles des différents groupes d'âge de truites ont évolué sous l'influence des deux facteurs antagonistes, température et densité de population, selon le type 

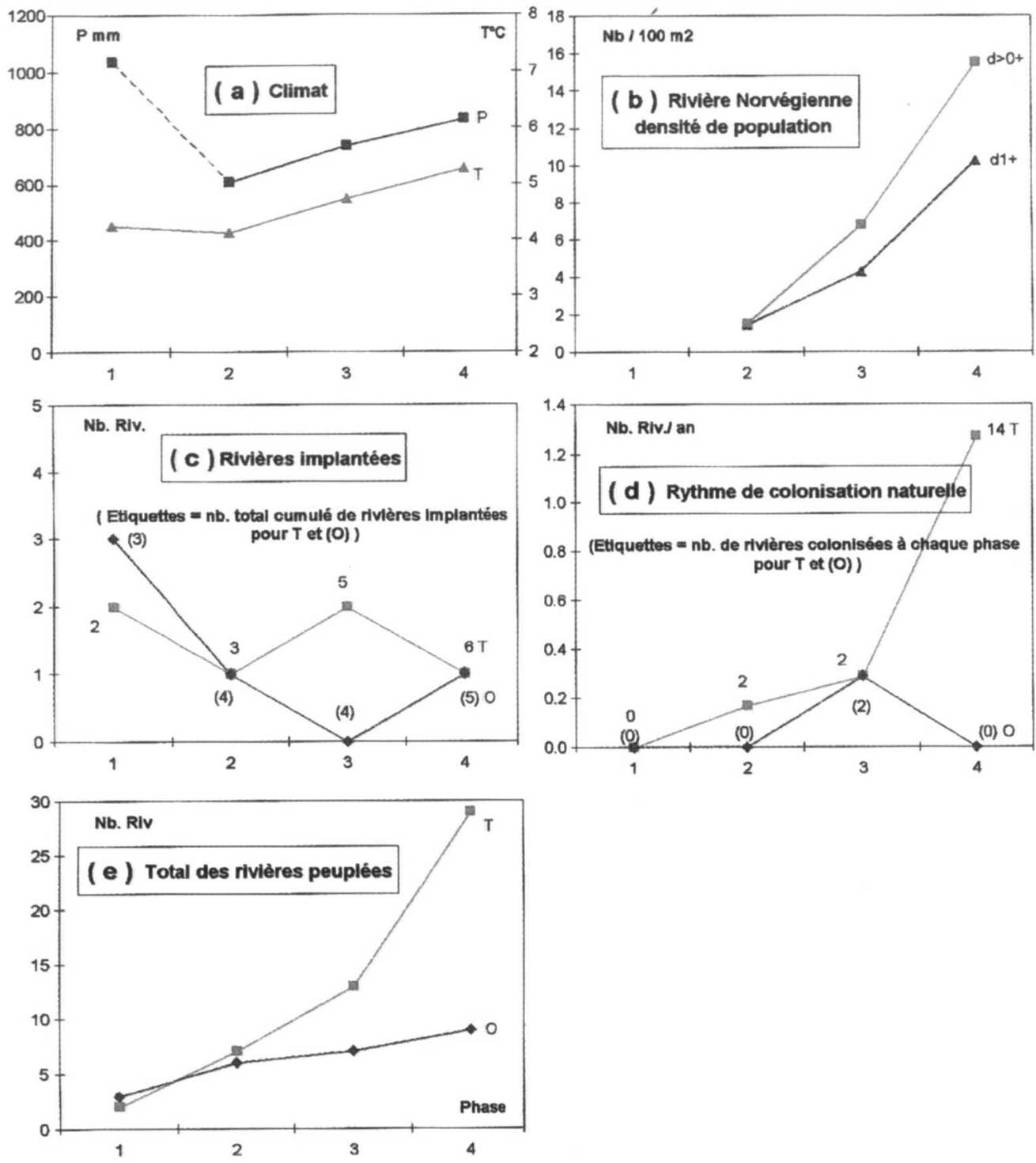

Figure 6

Evolutions constatées au cours des 4 phases $(1=1955-1962 ; 2=1963-1974 ; 3=1975-1981$; 4 = 1982-1992) définies pour les introductions :

a - Evolution de la température moyenne annuelle de l'air et des précipitations (Météo France, station de Port aux Français) : les valeurs des précipitations pendant la première phase sont sujettes à caution, n'ayant pas été recueillies dans les mêmes conditions qu'ultérieurement.

b - Evolution des densités de population estivale dans la rivière Norvégienne pour les truitelles d'un an et pour l'ensemble de la population (juvéniles et adultes sédentaires) à l'exception des alevins (moyennes des densités observées annuellement pendant chaque phase).

c - Nombre de rivières ayant été peuplées artificiellement de truites et d'ombles (introduction ou transfert) au cours de chaque phase. Le nombre cumulé de rivières peuplées en Truites $T$ ou en Ombles $(0)$ figure en étiquette (une même rivière peut recevoir l'une ou les deux espèces).

d - Nombre moyen annuel de rivières ayant été peuplées naturellement par des truites et des ombles venant de la mer et s'y étant reproduits, au cours de chacune des phases. Le nombre total par phase figure en étiquette.

e - Nombre total cumulé de rivières peuplées (artificiellement ou naturellement) en truites et en ombles, à la fin de chaque phase. 


\section{Figure 6}

Changes observed during the 4 phases of introductions $11=1955-1962 ; 2=1963-1974 ; 3=1975-$ $1981 ; 4$ = 1982-1992):

a - Mean annual air temperature and rainfall (Météo France, meteorological station of Port aux Français). Rainfall values during the first phase should be considered with caution because they were not collected under the same conditions as later on.

b - Summer population densities in the Norvégienne river for yearling trout and for the whole population (juveniles and sedentary adult fish) except fry (means of annually observed densities during each phase).

c - Number of streams planted with brown and brook trout (introduction or transplant) during each phase. The cumulative number of rivers stocked with brown $\mathrm{T}$ or brook $(O)$ trout is indicated in flags (any river can receive one or both species).

d - Mean annual number of streams naturally populated by brown and brook trout coming from the sea and effectively spawning, during each phase. Total number per phase is indicated in flag. e - Total cumulative number of rivers artificially or naturally populated with brown and brook trout, at the end of each phase.

d'habitat et la prédominance de l'un ou de l'autre : elles ont régulièrement augmenté lorsque l'effet des compétitions intraspécifiques était faible (1ère année de vie en rivière, 1er et 2ème été en mer), elles ont diminué dans les situations de compétition forte (3ème année en rivière) (DAVAINE et BEALL, 1992).

\subsubsection{Evolution inter-rivières}

Seuls les truites et les ombles de fontaine introduits dès la 1ère phase (de 1955 à 1962) ont colonisé spontanément d'autres rivières dont les embouchures étaient proches de leur rivière d'origine.

Si l'on excepte les ruisseaux de longueur inférieure au kilomètre et quelques petits étangs isolés, les ombles ont été introduits volontairement dans trois rivières pendant la 1ère phase, une petite rivière pendant la 2ème phase et un dernier réseau hydrographique en 4ème phase (Fig. 6c). Seulement deux rivières ont été colonisées naturellement pendant la 3ème phase, la rivière Norvégienne et la rivière des Albatros, à partir de la rivière du Château, dont l'embouchure n'est pas éloignée de plus de $5 \mathrm{~km}$ (Fig. 6d). Quelques ombles ont été contrôlés dans deux autres rivières sans qu'ils s'y soient reproduits. Cette souche issue d'une pisciculture française ne s'aventure en mer qu'exceptionnellement et pour de courtes périodes. L'aire de répartition de cette espèce à Kerguelen dépend étroitement des lieux de déversement et s'est stabilisée depuis plus de 10 ans, exception faite des poissons mis en élevage dans le lac d'Armor.

Les truites ont été introduites dans deux rivières en 1ère phase, une petite rivière en 2ème phase, deux autres rivières en 3ème phase et une en 4ème phase ; à l'exception des deux rivières de la 3ème phase, toutes les autres ont aussi accueilli des ombles (Tabl. I et Fig. 6c). En dépit d'une stratégie adaptative fondée sur l'exploitation du milieu marin, les truites sont restées au voisinage immédiat de l'estuaire des rivières ensemencées et $n$ 'ont colonisé naturellement que les rivières immédiatement voisines, soit deux rivières en 2ème phase et deux autres en 3ème phase (Fig. 6d). Pendant la 4ème phase, quatorze rivières ont été soudainement colonisées en l'espace de quelques années, comme le montrent les analyses de structure des populations régulièrement échantillonnées pendant cette période. Cet essaimage brutal et généralisé est à rapprocher de deux facteurs favorisants : d'une part, l'élévation progressive de la température moyenne, qui allonge la fenêtre salinité-température physiologiquement favorable aux truites en mer et qui a donc une action positive sur leur activité ; d'autre part, l'augmentation très sensible de la densité de population en rivière et, par conséquent, de l'effectif de truites migratrices susceptibles de s'aventurer toujours plus loin le long de la côte. C'est ainsi que la truite commune, ayant colonisé tous les cours d'eau ou parties de cours d'eau accessibles par la mer autour de la Péninsule Courbet (partie est des îles Kerguelen) et du Golfe 

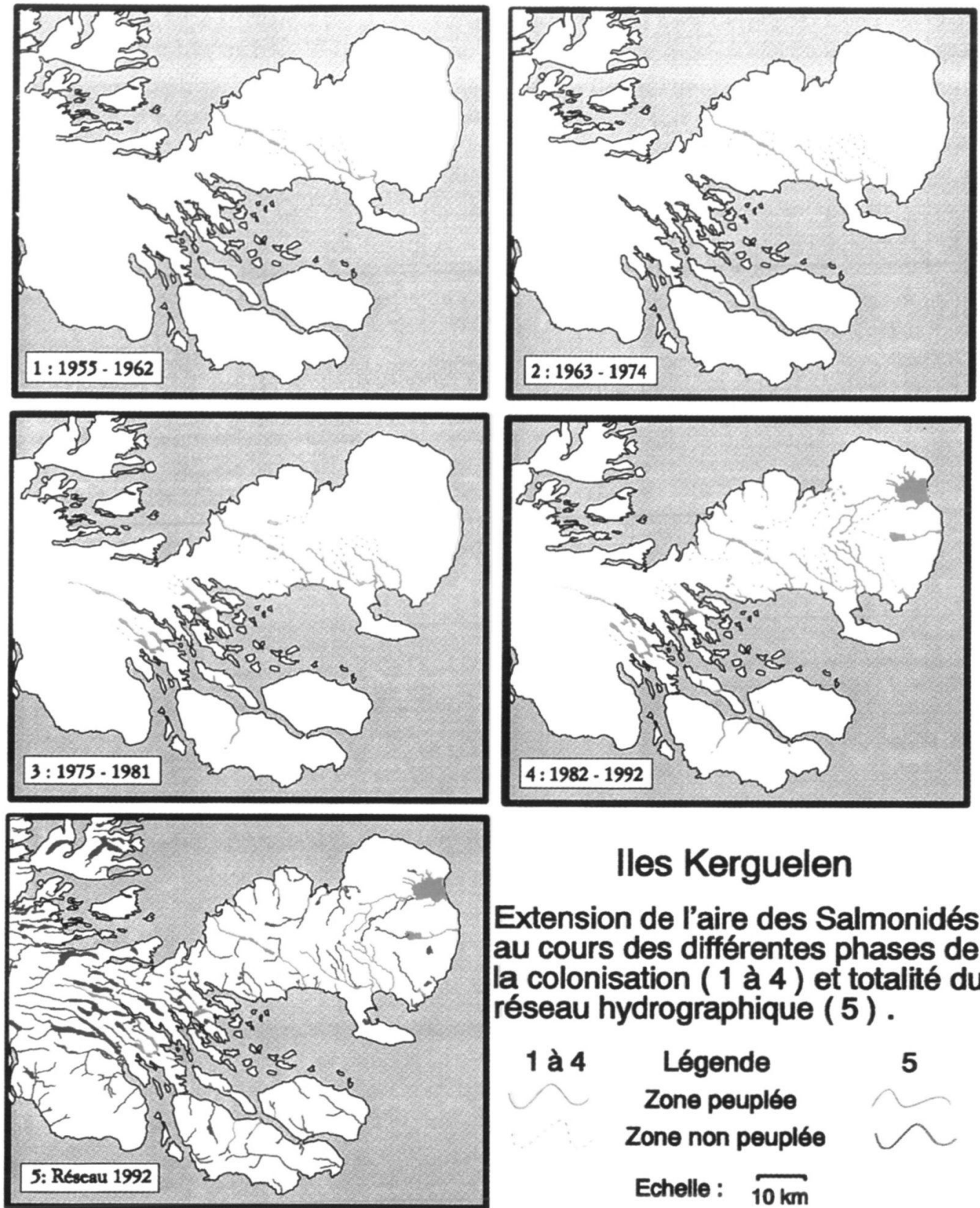

\section{Iles Kerguelen}

Extension de l'aire des Salmonidés au cours des différentes phases de la colonisation ( 1 à 4 ) et totalité du réseau hydrographique (5).

1 à 4

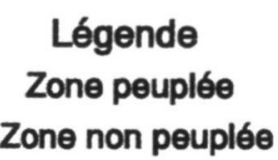

Echelle : $\overline{10 \mathrm{~km}}$

\section{Figure 7}

Cartes 1 à 4 - Extension de l'aire des Salmonidés à chaque phase de la colonisation (seules les rivières peuplées sont représentées et leurs parties amont restées vierges de poissons sont en pointillés).

Carte 5 - Ensemble du réseau hydrographique de la zone concernée (les parties peuplées figurent en rouge).

\section{Figure 7}

Maps 1 to 4 - Extension of Salmonids distribution area during each phase of colonization (only populated rivers are shown; dotted lines represent their upstream portions devoid of fish). Map 5 - The whole hydrographic system in the relevant zone (populated portions are in red). 
du Morbihan (partie sud-est), peuple actuellement une trentaine de rivières et lacs (Fig. 6e). La figure 7 illustre la stagnation des trois premières phases (Fig. 7-1 à 7-3) opposée à l'explosion colonisatrice de la quatrième (Fig. 7-4) et permet de relativiser les surfaces peuplées par rapport à celles beaucoup plus importantes restées vierges de poissons (Fig. 7-5).

Si l'évolution du climat régional vers un réchauffement se prolonge, les écosystèmes d'eau douce et marins deviendront de plus en plus favorables à la truite commune, qui pourrait avoir accès à toutes les parties de l'archipel. Cependant, la colonisation de l'ensemble des réseaux hydrographiques est impossible, sauf intervention humaine lourde, car des cascades infranchissables barrent l'accès à la majorité des rivières dès leur embouchure.

\section{PERSPECTIVES}

Entre les années 1950 et le début des années 1960, d'autres introductions de Salmonidés ont été réalisées dans des îles subantarctiques ou voisines. Aux îles Falkland, plus grandes que Kerguelen mais différant essentiellement par un environnement marin plus tempéré et une plus grande richesse faunistique, là aussi, après l'introduction de la truite commune et du saumon atlantique, seule la truite migratrice s'est naturalisée (ARROWSMITH et PENTELOW, 1965). Quelques truites arc-en-ciel furent libérées par une compagnie japonaise dans un réservoir artificiel de la Géorgie du Sud en 1964, dans des conditions si défavorables que pas une ne fut revue l'année suivante (HEADLAND, 1984). Enfin, à partir de truites communes déversées dans la rivière Van den Boogaard en 1964, sur l'île Marion, une petite population a survécu jusqu'en 1984 dans un secteur de quelques centaines de mètres, isolé de la mer, entre deux cascades infranchissables (COOPER et al., 1992). Les truites et ombles introduits dans l'île de la Possession (archipel de Crozet), en 1969, sont constamment au bord de l'extinction en raison d'un habitat moins favorable, malgré une faune aquatique plus riche qu'à Kerguelen (DAVAINE et BEALL, 1982a). Ces quelques exemples illustrent l'importance de la taille du réseau hydrographique, de la diversité des habitats et des possibilités de déplacements, dans le succès à long terme d'une introduction de Salmonidés jusqu'à l'établissement d'une métapopulation capable de composer avec l'instabilité d'un milieu à la limite des conditions tolérables pour l'espèce.

Si l'on se replace dans la perspective qui a motivé l'introduction des Salmonidés à Kerguelen, cette opération atteint pleinement son but : d'une part, quelques espèces sont bien naturalisées et procurent loisir et nourriture au personnel de la base; d'autre part, les connaissances biologiques acquises permettent de gérer rationnellement ces populations et de disposer de solutions d'intensification de la production dans des conditions connues, en cas de besoin et dans un contexte économique différent. Sur le plan scientifique, ces populations constituent des modèles extrêmement précieux pour des études très diverses, combinant les avantages de populations artificielles en dispositifs expérimentaux étroitement contrôlés et ceux de populations naturelles en environnement non perturbé (DAVAINE, 1989).

L'évolution moderne des mentalités tend à mettre, de plus en plus, la préservation de l'environnement au premier plan des préoccupations des gestionnaires, tandis que la reconstitution d'un environnement artificiel copiant le modèle métropolitain est considéré avec beaucoup de suspicion. Deux questions peuvent alors être posées. La première concerne d'abord les scientifiques : les Salmonidés introduits ont-ils un impact nuisible sur les écosystèmes subantarctiques? La seconde concerne l'administration : que faut-il faire des populations naturalisées?

\subsection{Impact des introductions}

Cette question a été particulièrement étudiée en raison de l'extraordinaire multiplication des introductions de Salmonidés depuis la fin du 19ème siècle, soit dans leur aire de répartition, quand une espèce recherchée est absente d'un écosystème, soit en dehors de leur aire de répartition. En général, les introductions intentionnelles de poissons ont rarement atteint leur objectif et ont été plutôt néfastes pour les espèces indigènes et les autres taxons, par le biais 
de la prédation, de la compétition, de l'hybridation ou de l'introduction de maladies (ALLENDORF, 1991). L'expérience montre que l'introduction d'une espèce de Salmonidé est d'abord néfaste pour d'autres espèces de Salmonidés en place, lorsque ces dernières ont des exigences écologiques (concept de niche) voisines tout en étant moins compétitives; de plus, elles peuvent s'hybrider avec l'espèce introduite et perdre leurs caractéristiques originales. C'est également néfaste pour d'autres espèces de poissons qui exploitent en commun une ressource limitée en quantité et en diversité (nourriture, habitat...). Les cas extrêmes concernent les acclimatations en dehors de l'aire de répartition, lorsque les espèces autochtones, poissons ou invertébrés de grande taille, constituent des proies particulièrement vulnérables quand il n'existe pas déjà de poissons prédateurs de grande taille auxquels ils puissent être adaptés ; c'est ainsi que la truite commune affecte durement certaines populations de Galaxias en Australie (AULT et WHITE, 1994), ou est rendue responsable de la quasi-disparition d'Anaspides tasmanias, Syncaridé de grande taille, dans les lacs de Tasmanie (WILLIAMS, 1974). Même si elle n'a pas d'effet spectaculaire, l'introduction ne peut pas être neutre et ses répercussions peuvent toucher tous les niveaux de l'écosystème : en Nouvelle-Zélande, FLECKER et TOWNSEND (1994) ont démontré que la densité et la biomasse d'insectes étaient inférieures dans les milieux avec truites que dans ceux sans poissons ou avec des Galaxiidés et, qu'inversement, la biomasse d'algues benthiques était supérieure suite à la raréfaction des insectes brouteurs. Lorsque des Salmonidés sont introduits dans des écosystèmes vierges de poissons, ils exercent alors une prédation sélective sur les plus grandes espèces d'invertébrés disponibles, allant jusqu'à la disparition des planctontes de grande taille dans les lacs (NILSSON, 1972; HARD, 1986; CHESS et al., 1993) ou bien provoquant l'émigration d'invertébrés, influencés chimiquement par la présence des truites, pour éviter la prédation (ANDERSEN et al., 1993).

Tous ces aspects ne concernent pas les rivières de Kerguelen, dont la faune aquatique peu diversifiée ne comporte que des espèces d'invertébrés abondantes, de petite taille et peu accessibles, et où les truites adultes se nourrissent essentiellement de faune terrestre entraînée accidentellement par le ruissellement. Le long du littoral, la prédation sur les Amphipodes marins est insignifiante par rapport à leur biomasse extraordinaire et l'impact des autres prédateurs. En revanche, les truites de mer importent dans les rivières une quantité non négligeable de matière organique d'origine marine, libérée sous forme d'ovules et de cadavres de géniteurs qui enrichissent l'écosystème dans des proportions mal connues, mais très semblables à celles des rivières à saumons de l'Arctique Pacifique ensemencées par les carcasses des géniteurs qui meurent tous après la ponte. On peut aussi mentionner la prédation sélective exercée par quelques individus isolés d'espèces d'oiseaux marins (sternes, cormorans, goélands) sur les juvéniles de truite et d'omble, sans que cela influence notablement le comportement alimentaire du reste de ces populations aviaires.

Signalons enfin que toutes les introductions ont été effectuées sous forme d'œufs, systématiquement désinfectés avant, pendant, et après leur transport, d'origine connue et sous le contrôle des services vétérinaires, afin de réduire autant que faire se peut, dans l'état de nos connaissances, les risques d'introduction de germes pathogènes.

\subsection{Gestion patrimoniale et avenir des Salmonidés}

L'Administration des Terres Australes possède un avantage considérable, qui est l'absence de population permanente dans les districts ; le poids des habitudes, des traditions, des intérêts à court terme d'un électorat local, n'existe pas et les décisions peuvent être prises rapidement, tout en prenant en compte l'état des connaissances constamment remis à jour par une communauté scientifique nombreuse. C'est ainsi que, dans l'esprit du Traité de l'Antarctique, des actions vigoureuses de protection d'espèces menacees et de restauration d'écosystèmes ont èté entreprises tout en y introduisant un suivi scientifique rigoureux.

Dans un district aussi vaste que Kerguelen, profondément marqué par la présence humaine (surexploitation des phoques et des manchots, introductions volontaires ou accidentelles d'espèces animales et végétales), il ne peut être question de revenir à un état 
antérieur d'écosystème vierge. Par contre, l'espace permet de protéger intégralement certaines zones d'habitat d'espèces sensibles et menacées, d'en réserver d'autres à des études sur la biologie des espèces si extraordinairement adaptées à cet environnement particulier, et enfin rend possible l'exercice d'autres activités humaines, en des lieux moins protégés, mais dans le respect des règles élémentaires de protection de l'environnement. Dans ces conditions, pourquoi ne pas conserver en l'état, par une réglementation adaptée, ces populations de Salmonidés, précieuses pour les recherches, limitées dans leur expansion et pratiquement inoffensives pour les espèces autochtones? Abandonnant toute idée d'exploitation intensive, le Territoire envisage de valoriser ce patrimoine biologique exceptionnel (peuplements denses de poissons âgés de grande taille), en organisant un tourisme halieutique sportif à l'instar de ce qui se pratique en Terre de Feu, Alaska, Groenland ou Kamtchatka. Cette activité, bien encadrée, sans conséquence notable pour l'environnement puisque limitée en nombre de participants et lieux fréquentés, inoffensive pour les poissons (les prélèvements étant réduits au minimum, les autres prises étant remises à l'eau), viendrait compléter le tourisme de croisière et découverte de la nature ainsi que le tourisme de randonnée naturaliste, déjà expérimentés avec succès. Ce territoire extraordinaire, réservé jusqu'alors aux quelques membres des missions, pourrait s'ouvrir à un plus large public respectueux de la nature.

\section{REMERCIEMENTS}

Nous souhaitons remercier l'administration des TAAF (Terres Australes et Antarctiques Françaises) et plus particulièrement ses responsables scientifiques successifs : J.P. BLOCH et B. MORLET, ainsi que R. GENDRIN (Directeur de l'Institut Polaire), pour nous avoir donné la possibilité de travailler dans le long terme aux îles Kerguelen en nous fournissant le support logistique et financier.

De nombreux membres des missions australes, qui ne peuvent tous être cités ici, ont participé aux observations.

Les opérations de terrain ont pu s'effectuer au mieux dans un contexte difficile, grâce au soutien et à l'efficacité de A. LAMALLE, responsable de la logistique et des opérations scientifiques. Le traitement des données est également redevable à D. BAZIN et Y. DAVAINE, et la frappe du manuscrit à M.C. CAZENAVE.

\section{BIBLIOGRAPHIE}

ALLENDORF F.W., 1991. Ecological and genetic effects of fish introductions : synthesis and recommendations. Can. J. Fish. Aquat. Sci., 48 (Suppl.1), 178-181.

ANDERSEN T.H., FRIBERG N., HANSEN H.O., IVERSEN T.M., JACOBSEN D., KROJGAARD L., 1993. The effects of introduction of brown trout (Salmo trutta L.) on Gammarus pulex L. drift and density in two fishless Danish streams. Arch. Hydrobiol., 126, 3, 361-371.

ARROWSMITH E., PENTELOW F.T.K., 1965. The introduction of trout and salmon to the Falkland Islands. Salm. Trout Mag., 174, 119-129.

AULT T.R., WHITE R.W.G., 1994. Effects of habitat structure and the presence of brown trout on the population density of Galaxias truttaceus in Tasmania, Australia. Trans. Am. Fish. Soc., 123, 939-949.

BARBAULT R., BLANDIN P., 1979. La notion de stratégie adaptative : sur quelques aspects énergétiques, démographiques et synécologiques. In BARBAULT R., BLANDIN P. et MEYER J.A., eds., Problèmes d'Ecologie théorique : les stratégies adaptatives, Maloine, Paris, 1-27.

CHESS D.W., GIBSON F., SCHOLZ A.T., WHITE R.J., 1993. The introduction of Lahontan cutthroat trout into a previously fishless lake: feeding habits and effects upon the zooplankton and benthic community. Jour. Freshwat. Ecology, 8, 3, 215-225. 
CHOWN S.L., SMITH V.R., 1993. Climate change and the short-term impact of feral house mice at the sub-Antarctic Prince Edward Islands. Oecologia, 96, 508-516.

COOPER J., CRAFFORD J.E., HECHT T., 1992. Introduction and extinction of brown trout (Salmo trutta L.) in an impoverished subantarctic stream. Antarctic Science, 4 (1), 9-14.

DAVAINE P., 1988. Espèces de Salmonidés introduites, perspectives économiques. Actes du Colloque sur la Recherche française dans les terres Australes, Strasbourg 1987, 255-267.

DAVAINE P., 1989. Hydrobiologie. In DUCHENE J.C., Kerguelen, Recherches au bout du monde, TAAF, Mission de Recherche, 325-334.

DAVAINE P., 1991. Sea ranching of coho salmon in the Kerguelen islands (TAAF). ICES Ana. Cat. Fish. Com., M: 20, 10 p. +6 fig., 6 tab.

DAVAINE P., BEALL E., 1982 a. Introduction de Salmonidés dans les Terres Australes et Antarctiques Françaises. CNFRA, 51, 289-300.

DAVAINE P., BEALL E., 1982 b. Acclimatation de la truite commune, Salmo trutta L., en milieu subantarctique (îles Kerguelen). II - Stratégie adaptative. CNFRA, 51, 399-411.

DAVAINE P., BEALL E., 1992. Relationships between temperature, population density and growth in a seatrout population (S. trutta L.) of the Kerguelen islands. ICES J. Mar. Sci., 49, 445-451.

DELÉPINE G., 1995. Les îles Australes Françaises. Ed. Ouest-France, Rennes, 213 p.

ELLIOTT J.M., 1994. Quantitative Ecology and the brown trout. Oxford University Press, Oxford, $286 \mathrm{p}$.

FLECKER A.S., TOWNSEND C.R., 1994. Community-wide consequences of trout introduction in New Zealand streams. Ecological Applications, 4, 4, 798-807.

GUYOMARD R., GRÉVISSE C., OURY F.X., DAVAINE P., 1984. Evolution de la variabilité génétique inter et intra-populations de populations de Salmonidés issues de mêmes pools géniques. Can. J. Fish. Aquat. Sci., 41, 7, 1024-1029.

HARD J.J., 1986. Production and yield of juvenile chinook salmon in two Alaskan lakes. Trans. Am. Fish. Soc., 115, 305-313.

HEADLAND R., 1984. The Island of South Georgia. Cambridge University Press, Cambridge, $293 \mathrm{p}$.

LÉSEL R., THÉRÉZIEN Y., VIBERT R., 1971. Introduction de Salmonidés aux îles Kerguelen. I Premiers résultats et observations préliminaires. Ann. Hydrobiol., 2, 2, 275-304.

NILSSON N.A., 1972. Effects of introductions of Salmonids into barren lakes. J. Fish. Res. Bd Canada, 29, 6, 693-697.

RICHERT X., 1958. $Y$ aura-t-il des truites aux iles Kerguelen ? TAAF, 4/5, 4-9.

SALINGER M.J., 1982. On the suggestion of post 1950 warming over New Zealand. New Zealand Journal of Science, 25, 77-86.

THOMAS T., DAVAINE P., BEALL E., 1981. Dynamique de la migration et reproduction de la truite de mer, Salmo trutta L., dans la rivière Norvégienne, îles Kerguelen, TAAF. CNFRA, $47,5-42$.

THORPE J.E., 1980. Salmon ranching. Academic Press, London, $441 \mathrm{p}$.

WILLIAMS W.D., 1974. Freshwater crustacea. In WILLIAMS W.D., ed., Biogeography and Ecology in Tasmania, Junk, the Hague, 63-112.

WOJTENKA J., VANSTEENBERGHE F., 1982. Variations nycthémérales et saisonnières de la faune en place et en dérive, stratégie alimentaire de la truite (Salmo trutta L.) dans une petite rivière des îles Kerguelen. CNFRA, 51, 413-423.

YOUNG E.C., 1991. Critical ecosystems and nature conservation in Antarctica. In HARRIS C.M. and STONEHOUSE B., eds., Antarctica and global climatic change, Belhaven Press, London, 117-146. 